\title{
Long-Term Molecular Remission Achieved by Antibody Anti-CD22 and Ponatinib in a Patient Affected by Ph'+ Acute Lymphoblastic Leukemia Relapsed after Second Allogeneic Hematopoietic Stem Cell Transplantation: A Case Report
}

\author{
Maria Cristina Pirosa ${ }^{a}$ Salvatore Leotta ${ }^{a}$ Alessandra Cupri $^{a}$ Stefania Stella $^{b}$ \\ Enrica Antonia Martino ${ }^{a}$ Luca Scalise $^{a}$ Giuseppe Sapienza $^{a}$ Valeria Calafiore $^{a}$ Elisa Mauro $^{a}$ \\ Andrea Spadaro $^{a}$ Paolo Vigneri $^{\mathrm{b}}$ Francesco Di Raimondo $^{\mathrm{a}}$ Giuseppe Milone ${ }^{\mathrm{a}}$ \\ ${ }^{a}$ Division of Hematology and BMT - Ospedale Policlinico, Catania, Italy; ${ }^{\mathrm{b}}$ Department of Clinical and Experimental \\ Medicine, University of Catania, Catania, Italy
}

\section{Established Facts}

- The prognosis of acute lymphoblastic leukemia following early relapse after allogeneic bone marrow transplantation is very poor. Results of salvage strategies [chemotherapy, donor lymphocyte infusions (DLI), second transplant] are very disappointing in most cases.

\section{Novel Insights}

- New drugs, such as monoclonal antibodies and 3rd generation tyrosine kinase inhibitors, offer encouraging therapeutic options, but up to now no experience/reports have been published on their use in salvage treatment in combination with DLI as in our case report.

\section{Keywords}

Acute lymphoblastic leukemia $\cdot$ Stem cell transplantation . Inotuzumab · Ponatinib

\begin{abstract}
$\mathrm{Ph}^{\prime}+$ acute lymphoblastic leukemia (Ph'+-ALL) is an oncohematologic disorder for which allogeneic bone marrow transplantation still offers the only chance of cure. However, relapse is the main reason for treatment failure, also after hematopoietic stem cell transplantation (HSCT). New drugs, such as third generation tyrosine kinase inhibitors (TKIs) and
\end{abstract}

monoclonal antibodies, have expanded the therapeutic landscape, especially in patients who relapsed before HSCT. Very few reports, up to now, have described the use of both classes of these new agents in combination with donor lymphocyte infusions (DLI) in the setting of patients who relapsed after HSCT. We report on a young patient affected by $\mathrm{Ph}^{\prime}+-\mathrm{ALL}$, who relapsed after the second HSCT and who reached molecular remission and long-term disease control by treatment with the anti-CD22 monoclonal antibody inotuzumab ozogamicin, DLI, and the 3rd generation TKI ponatinib.

\section{KARGER}

(c) 2018 S. Karger AG, Basel

E-Mail karger@karger.com

www.karger.com/che
Salvatore Leotta

Division of Hematology and BMT - Ospedale Policlinico

Via S. Sofia 78

IT-95124 Catania (Italy)

E-Mail leotta3@yahoo.it 


\section{Introduction}

Allogeneic hematopoietic stem cell transplantation (HSCT) still remains the standard of cure for Ph'+ acute lymphoblastic leukemia (Ph'+-ALL) $[1,2]$ even though, in the last decade, the introduction to the therapeutic scenario of tyrosine kinase inhibitors (TKIs) has improved the chance of survival [2-4].

However, even after the development of TKIs, relapse remains an important reason for treatment failure [5]. The prognosis of patients who relapse immediately after HSCT is extremely poor, and the median 2-year survival ranges between 10 and 16\% [6]. New drugs for relapsed patients include monoclonal antibodies and 3rd generation TKIs $[7,8]$.

In the present case report, we describe our experience with a young patient affected by Ph'+ ALL relapsed after the second HSCT who obtained and maintained a complete remission after treatment with inotuzumab ozogamicin (IO) and ponatinib.

\section{Case Report}

We present the case of a 23-year-old female who was referred to our institute in December 2011 for asthenia and an abnormal CBC: anemia (Hb $6.5 \mathrm{~g} / \mathrm{dL})$, thrombocytopenia $\left(14,000 / \mathrm{mm}^{3}\right)$, and leukocytosis (WBC: $13,940 / \mathrm{mm}^{3}$ ) with absolute lymphocytosis $(80 \%)$. The morphological exam showed 60 and $80 \%$ of lymphoid blast cells on the bone marrow and peripheral blood, respectively.

Immunophenotype confirmed the diagnosis of B-Cell ALL (CD19+, CD10+, HLA-DR+, CD20+/-, cyCD79a+, CD58+, $\mathrm{TdT}+, \mathrm{CD} 34+$, and $\mathrm{CD} 33+/-)$. Conventional cytogenetics revealed the presence of the Ph' chromosome in all examined metaphases with no further abnormalities (karyotype 46, XX, t[9;22]). Multiplex PCR showed a b3a2 BCR-ABL transcript with BCRABL/ABL levels of $166.17 \%$ measured by real-time PCR (Q-PCR) [9]. In addition, despite the negative multiplex PCR, the patient displayed ela2 BCR-ABL transcript with BCR-ABL/ABL levels of $0.74872 \%$ measured by Q-PCR.

The patient was assigned to the GIMEMA LAL 1509 protocol [10]. Before treatment, approval by the Institutional Ethics Committee and written informed consent by the patient were obtained. According to the protocol, the patient was treated with dasatinib $140 \mathrm{mg}$ q.d. from day +1 to day +84 and a prednisone dose escalation up to $60 \mathrm{mg} / \mathrm{m}^{2}$ as a pretreatment from day -6 to day -3 , continuing to day +24 and then tapered from day +25 to day +32 . CNS prophylaxis was performed with intrathecal methotrexate $15 \mathrm{mg}$ and 6-methylprednisolone $20 \mathrm{mg}$, initially biweekly and then monthly, for a total of 12 lumbar punctures. The patient achieved a deep molecular response with BCR-ABL/ABL transcript levels of $0.0014 \%$ after the induction and, according to the protocol, was started on dasatinib $140 \mathrm{mg}$ q.d. as maintenance.

In November 2012, the BCR-ABL/ABL transcript level increased from 0.0014 to $0.0122 \%$. Thus, the patient continued da- satinib until January 2013 when she underwent HSCT from HLAidentical sibling. The myeloablative conditioning regimen consisted of busilvex $3.2 \mathrm{mg} / \mathrm{kg}$ and fludarabine $40 \mathrm{mg} / \mathrm{m}^{2}$ for 4 days. The stem cells dose was CD $34+2 \times 10^{6} / \mathrm{kg}$. The patient developed acute grade 2 GVHD responsive to steroid therapy.

In February 2013, a bone marrow post-transplantation investigation of BCR-ABL/ABL levels evidenced deep molecular response (BCR-ABL/ABL $=0.00000 \%$ with 33,786 copies of $A B L)$.

In November 2013, hematological relapse occurred (blast cells $20 \%$ of total nucleated cells on bone marrow smear), and the patient underwent salvage chemotherapy according to the scheme L-VAMP with methotrexate $1,000 \mathrm{mg} / \mathrm{m}^{2}$, vincristine $1.4 \mathrm{mg} / \mathrm{m}^{2}$, cytarabine $500 \mathrm{mg} / \mathrm{m}^{2}$ on day +1 and dexamethasone $20 \mathrm{mg}$ from day +1 to +5 . Revaluation after chemotherapy showed persistent residual disease (bone marrow blasts on immunophenotype 1\%). For a disease for which allogeneic transplantation still represents the standard of care, a second transplant from an unrelated donor seemed the best choice for the patient to get a better chance of healing. With the intention of preparing the patient for transplant with the lowest possible level of disease, we chose imatinib as "bridgeto-transplant therapy" because the disease had proved to be resistant to dasatinib, and ponatinib had not been approved by the Institutional Italian Drug Agency in 2014. For these reasons, in December 2014, the patient began a maintenance therapy with imatinib $600 \mathrm{mg} / \mathrm{day}$, and a workup for a second transplant from an unrelated donor was started.

The second HSCT by an 8/8-matched unrelated donor was performed in February 2014 by using reduced intensity conditioning (fludarabine $25 \mathrm{mg} / \mathrm{m}^{2}$ for 5 days and melphalan $70 \mathrm{mg} / \mathrm{m}^{2}$ for 2 days) and mobilized peripheral blood stem cells (CD34+4.1 × 106\% $\mathrm{kg}$ ). The patient developed acute GVHD overall grade 2 (skin and bowel) responsive to steroids.

Before the transplant, in January 2014, our patient achieved a major molecular response with BCR-ABL/ABL $=0.00925 \%$, and 120 days after transplant, she was started on a maintenance therapy with imatinib $800 \mathrm{mg}$ daily. In September 2014, a bone marrow assessment showed the loss of molecular response (BCR-ABL/ $\mathrm{ABL}=0.24776 \%)$. Mutational analysis of the BCR-ABL tyrosine kinase domain after cloning of the PCR amplification product displayed a previously described insertion of 35 nucleotides from ABL intron 8 at the normal junction between exons 8 and 9. This insertion forms a stop codon after 10 intron-encoded amino acids [11] that, in CML progressing to lymphoid blast crisis, has been associated with resistance to imatinib and dasatinib [12].

Therapy with nilotinib $800 \mathrm{mg} /$ day was started and maintained until February 2015, when a hematological relapse occurred (WBC $37,000 / \mathrm{mm}^{3}$, with $80 \%$ of lymphoid blast cells on peripheral blood). Then, our patient was started on a salvage therapy with 3 doses of vincristine $1.5 \mathrm{mg} / \mathrm{m}^{2}$ between March and April 2015. In May 2015, she began IO therapy which was administered according to the schedule: $0.8 \mathrm{mg} / \mathrm{m}^{2}$ i.v. on day +1 and $0.5 \mathrm{mg} / \mathrm{m}^{2}$ i.v. on days +8 and +15 as described by Kantarjian et al. [13].

Between May and July 2015, 3 cycles of treatment were performed. On May 2015, before administration of IO, revaluation of disease showed $50 \%$ of blast cells on the bone marrow. In addition, the patient was leukopenic (WBC: $\left.2,730 / \mathrm{mm}^{3}\right)$, anemic ( $\mathrm{Hb} 8.7 \mathrm{~g} /$ $\mathrm{dL}$ ), and thrombocytopenic (PLT 29,000/ $\mathrm{mm}^{3}$ ). In June 2015, after the first cycle of IO, a deep molecular response was achieved (BCR$\mathrm{ABL} / \mathrm{ABL}=0.00000 \%$ with 138,976 copies of $\mathrm{ABL})$. The response was maintained after the end of IO treatment, both on the bone 


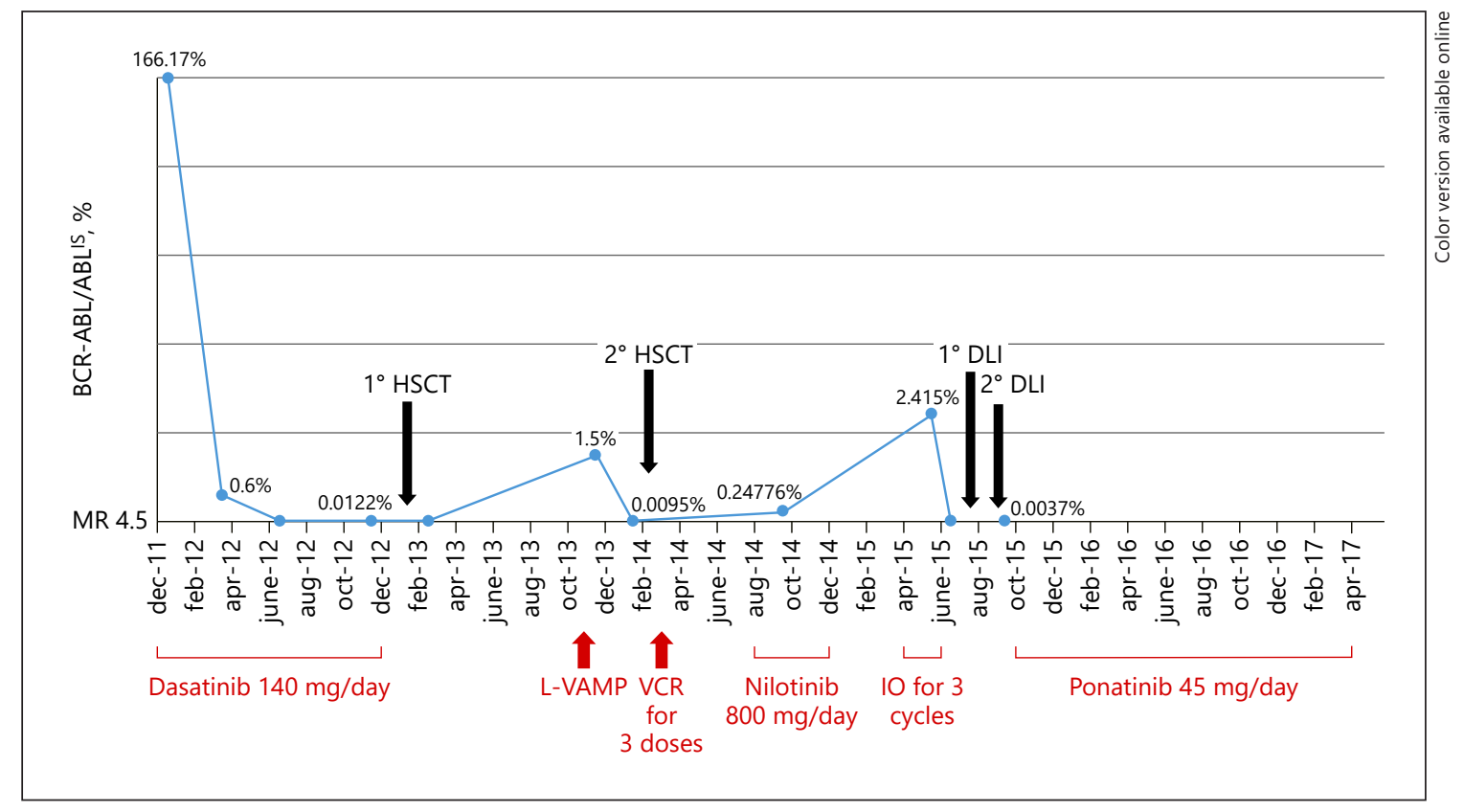

Fig. 1. Clinical course of disease and treatment strategies employed. ALL relapsed after the first and second HSCT despite deep molecular response. After therapy with IO, DLI, and ponatinib, the patient is still alive and has maintained a deep molecular response for the last 2 years. MR, molecular response.

marrow and on peripheral blood. During treatment, we recorded a grade 3 WHO hematological toxicity (asymptomatic thrombocytopenia $<50,000 / \mathrm{mm}^{3}$ ) which did not induce suspension of therapy and resolved spontaneously after stopping IO treatment. In July 2015, a first donor leukocyte infusion (DLI) was performed $\left(\mathrm{CD} 3+3.3 \times 10^{6} / \mathrm{kg}\right)$. After 30 days, a new DLI was carried out $\left(\mathrm{CD} 3+1 \times 10^{7} / \mathrm{kg}\right)$. For DLI, no immunosuppressive prophylaxis was administered.

After the second DLI, in August 2015, liver function indexes increased with ALT and ALP reaching up to 5 times the upper normal limit and GGT 7 times the upper normal limit. Microbiological investigations excluded infectious hepatitis, and the clinical diagnostic criteria of VOD were absent [14]. Signs of GVHD were not observed at any other organ. The patient was asymptomatic and refused a liver biopsy. She received therapy with ursodeoxycholic acid and detoxifying agents (silimarin) until November 2015 when liver indexes completely normalized. Normalization of $\mathrm{CBC}\left(\mathrm{Hb}>12 \mathrm{~g} / \mathrm{dL}, \mathrm{PLT}>100,000 / \mathrm{mm}^{3}, \mathrm{WBC}>4,000 / \mathrm{mm}^{3}\right.$, and neutrophils $>1,000 / \mathrm{mm}^{3}$ ) and deep molecular response (BCR/ ABL levels 0.0037\%) were maintained until September 2015 when the patient was started on ponatinib ( $45 \mathrm{mg} /$ day). Low dose acetylsalicylic acid (100 mg/day) was added as prophylaxis for cardiovascular events.

Currently, ponatinib therapy is still continuing, and the patient maintains deep molecular response (last evaluation performed in May 2018 with BCR-ABL/ABL $=0.00000 \%)$. No adverse effects of clinical significance were observed. From the start of treatment, no toxicity related to ponatinib was registered, and the patient is off any other drug that might interact with ponatinib.

\section{Discussion}

Allogeneic HSCT is currently the only available cure for adult patients affected by ALL. Unfortunately, relapse represents the major reason for treatment failure [15]. In most cases, the prognosis of patients who relapse after transplantation is very poor, especially for early relapses (less than 6 months from the transplant) $[6,16]$, and therapeutic options for these patients are extremely limited.

Up to now, conventional therapeutic approaches (suspension of immunosuppressive therapy, DLI, chemotherapy, and retransplantation) are burdened by a high percentage of failure due to toxicity or relapses [16-18]. New drugs, such as monoclonal antibodies or third generation TKIs, offer new possibilities for treatment of these patients. Few clinical experiences published so far evaluate the use of these drugs in the "posttransplant" setting $[19,20]$. We described the case of a patient affected by Ph'+ ALL following early relapse after a second HSCT, who was treated with a combination of IO, DLI, and ponatinib. After starting therapy, the patient quickly achieved deep molecular response and still maintains this response after 3 years. The treatment did not require hospitalization, and there was no toxicity that might have led to discontinuation or reduction of therapy, allowing a satisfying quality of life. 
We think that, considering the highly aggressive nature of the disease (refractoriness to chemotherapy and to first as well as second generation TKIs and early relapse after transplant), the clinical course of our patient would have been worse using conventional treatment. Our therapeutic approach was based on the use of a monoclonal antibody selective for B lymphocytes and the use of a TKI whose activity against resistant clones is well documented [21]. IO has already been shown to induce a quick and complete hematologic and molecular remission in patients suffering from relapsed/refractory ALL $[13,22]$. In a large phase 3 randomized study [23], inotuzumab did better than conventional chemotherapy in the percentage of complete remission and in the depth of such remission. However, the duration of response was only of a few months (4.6 vs. 3.1 months compared to conventional chemotherapy) and also the progression-free survival was short ( 5 vs. 1.8 months). A total of $40 \%$ of patients treated with inotuzumab could proceed to HSCT compared to $11 \%$ of patients treated with chemotherapy [23].

No study has been published so far on the use of IO in the setting of leukemia relapse after HSCT as a bridge to DLI and in addition to ponatinib. In our experience, IO allowed a rapid induction of deep molecular response. Moreover, molecular response indicates a high level of depth of response rendering the disease undetectable, but it does not give certainty that the leukemic clone has been eradicated. Deep molecular response in ALL is the main prognostic factor predicting a disease-free survival, but some patients also relapse after HSCT performed under MRD-negative conditions [24]. Our patient's history reveals that the disease relapsed despite achieving deep molecular response before both HSCTs and DLI (Fig. 1). These considerations prompted us to start also treatment with TKIs. Considering the refractoriness to first and second generation TKI, we chose to use ponatinib, which has also shown an effect on leukemic microenvironment in vitro as well as on the blast cell [25]. Up to now, this strategy has extended remission to 3 years after relapse.

Treatment of relapse after HSCT also included the use of DLI and nowadays, in the post-transplant setting, new drugs can be used as a bridge to DLI. At the current time, there is little published experience on the use of monoclonal antibodies as bridge to DLI. Ueda et al. [26] described 4 cases of ALL relapsed after HSCT treated with blinatumomab (2-4 cycles) and DLI obtaining a durable response (7-13 months) in 2 of 4 patients. In their experience, this approach was feasible and safe. There have, up to now, been no published data on the use of IO as bridge to DLI. The response to DLI alone of ALL relapses after HSCT is very disappointing with the overall 2-year survival not exceeding $10 \%$ [17].

Data reporting on the prophylactic use of DLI performed in high-risk ALL provide better results [27, 28]. Most likely, the use of adoptive immunotherapy in conditions of minimal disease is more effective than in overt relapse.

In the new therapeutic scenario, monoclonal antibodies could be used to reduce leukemic burden to minimum levels to increase sensitivity to DLI. The use of third generation TKIs, nevertheless, could play an important role in the continuing therapy, with the purpose of extending disease-free survival in a challenging clinical situation such as ALL relapse after bone marrow transplantation.

\section{Statement of Ethics}

The authors have no ethical conflicts to declare.

\section{Disclosure Statement}

The authors have no conflicts of interest to disclose.

\section{References}

1 Sureda A, Bader P, Cesaro S, Dreger P, Duarte $\mathrm{RF}$, Dufour C, et al. Indications for allo- and auto-SCT for haematological diseases, solid tumours and immune disorders: current practice in Europe, 2015. Bone Marrow Transplant. 2015 Aug;50(8):1037-56.

2 Chiaretti S, Foà R. Management of adult Phpositive acute lymphoblastic leukemia. $\mathrm{He}$ matology (Am Soc Hematol Educ Program). 2015;2015(1):406-13.
3 Mizuta S, Matsuo K, Nishiwaki S, Imai K, Kanamori H, Ohashi K, et al. Pretransplant administration of imatinib for allo-HSCT in patients with BCR-ABL-positive acute lymphoblastic leukemia. Blood. 2014 Apr; 123(15):2325-32.

4 Brissot E, Labopin M, Beckers MM, Socié G, Rambaldi A, Volin L, et al. Tyrosine kinase inhibitors improve long-term outcome of allogeneic hematopoietic stem cell transplantation for adult patients with Philadelphia chromosome positive acute lymphoblastic leukemia. Haematologica. 2015 Mar;100(3):392-9.
5 Pfeifer H, Wassmann B, Bethge W, Dengler I, Bornhäuser M, Stadler M, et al.; GMALL Study Group. Randomized comparison of prophylactic and minimal residual diseasetriggered imatinib after allogeneic stem cell transplantation for BCR-ABL1-positive acute lymphoblastic leukemia. Leukemia. 2013 Jun; 27(6):1254-62
Long-Term Remission Achieved by Anti-CD22 and Ponatinib in Ph'+-ALL
Chemotherapy 2018;63:220-224

DOI: $10.1159 / 000492941$ 
6 Spyridonidis A, Labopin M, Schmid C, Volin L, Yakoub-Agha I, Stadler M, et al.; Immunotherapy Subcommittee of Acute Leukemia Working Party. Outcomes and prognostic factors of adults with acute lymphoblastic leukemia who relapse after allogeneic hematopoietic cell transplantation. An analysis on behalf of the Acute Leukemia Working Party of EBMT. Leukemia. 2012 Jun;26(6):1211-7.

7 Cortes JE, Kim DW, Pinilla-Ibarz J, le Coutre P, Paquette R, Chuah C, et al.; PACE Investigators. A phase 2 trial of ponatinib in Philadelphia chromosome-positive leukemias. N Engl J Med. 2013 Nov;369(19):1783-96.

8 Jabbour E, O’Brien S, Ravandi F, Kantarjian $\mathrm{H}$. Monoclonal antibodies in acute lymphoblastic leukemia. Blood. 2015 Jun;125(26): 4010-6.

9 Baccarani M, Deininger MW, Rosti G, Hochhaus A, Soverini S, Apperley JF, et al. European LeukemiaNet recommendations for the management of chronic myeloid leukemia: 2013. Blood. 2013 Aug;122(6):872-84.

10 Chiaretti S, Vitale A, Elia L, Albino S, Piciocchi A, Fazi P et al. First Results of the Multicenter Total Therapy Gimema LAL 1509 Protocol for De Novo Adult Philadelphia Chromosome Positive $(\mathrm{Ph}+)$ Acute Lymphoblastic Leukemia (ALL) Patients. Blood. 2014;124: 797.

11 Laudadio J, Deininger MW, Mauro MJ, Druker BJ, Press RD. An intron-derived insertion/truncation mutation in the BCR-ABL kinase domain in chronic myeloid leukemia patients undergoing kinase inhibitor therapy. J Mol Diagn. 2008 Mar;10(2):177-80.

12 Stagno F, Stella S, Berretta S, Massimino M, Antolino A, Giustolisi R, et al. Sequential mutations causing resistance to both Imatinib Mesylate and Dasatinib in a chronic myeloid leukaemia patient progressing to lymphoid blast crisis. Leuk Res. 2008 Apr;32(4):673-4.

13 Kantarjian H, Thomas D, Jorgensen J, Kebriaei $\mathrm{P}$, Jabbour E, Rytting M, et al. Results of inotuzumab ozogamicin, a CD22 monoclonal antibody, in refractory and relapsed acute lymphocytic leukemia. Cancer. 2013 Aug; 119(15):2728-36.
14 Mohty M, Malard F, Abecassis M, Aerts E, Alaskar AS, Aljurf M, et al. Revised diagnosis and severity criteria for sinusoidal obstruction syndrome/veno-occlusive disease in adult patients: a new classification from the European Society for Blood and Marrow Transplantation. Bone Marrow Transplant. $2016 \mathrm{Jul} ; 51(7): 906-12$.

15 Fielding AK, Rowe JM, Richards SM, Buck G, Moorman AV, Durrant IJ, et al. Prospective outcome data on 267 unselected adult patients with Philadelphia chromosome-positive acute lymphoblastic leukemia confirms superiority of allogeneic transplantation over chemotherapy in the pre-imatinib era: results from the International ALL Trial MRC UKALLXII/ECOG2993. Blood. 2009 May; 113(19):4489-96

16 Poon LM, Hamdi A, Saliba R, Rondon G, Ledesma C, Kendrick M, et al. Outcomes of adults with acute lymphoblastic leukemia relapsing after allogeneic hematopoietic stem cell transplantation. Biol Blood Marrow Transplant. 2013 Jul;19(7):1059-64.

17 Collins RH Jr, Goldstein S, Giralt S, Levine J, Porter D, Drobyski W, et al. Donor leukocyte infusions in acute lymphocytic leukemia. Bone Marrow Transplant. 2000 Sep;26(5): 511-6.

18 McIver ZA, Yin F, Hughes T, Battiwalla M, Ito S, Koklanaris E, et al. Second Hematopoietic Stem Cell Transplantation for Leukemia Relapsing after Myeloablative T Cell Depleted Transplants Does Not Prolong Survival. Bone Marrow Transplant. 2013 Sep;48(9):1192-7.

19 Renzi D, Marchesi F, De Angelis G, Elia L, Salvatorelli E, Gumenyuk S, et al.; Rome Transplant Network. Ponatinib Induces a Persistent Molecular Response and Graft-versus-Host Disease/Graft-versus-Leukemia Effect in a Patient with Philadelphia-Positive Acute Lymphoblastic Leukemia with a T315I Mutation following Early Relapse after Allogeneic Transplant. Chemotherapy. 2017; 62(1):58-61.

20 Assi R, Kantarjian H, Short NJ, Daver N, Takahashi K, Garcia-Manero G, et al. Safety and Efficacy of Blinatumomab in Combination With a Tyrosine Kinase Inhibitor for the Treatment of Relapsed Philadelphia Chromosome-positive Leukemia. Clin Lymphoma Myeloma Leuk. 2017 Dec;17(12):897-901.
21 Miller GD, Bruno BJ, Lim CS. Resistant mutations in CML and $\mathrm{Ph}(+) \mathrm{ALL}$ - role of ponatinib. Biologics. 2014 Oct;8:243-54.

22 Kantarjian H, Thomas D, Jorgensen J, Jabbour E, Kebriaei P, Rytting M, et al. Inotuzumab ozogamicin, an anti-CD22-calecheamicin conjugate, for refractory and relapsed acute lymphocytic leukaemia: a phase 2 study. Lancet Oncol. 2012 Apr;13(4):40311.

23 Kantarjian HM, DeAngelo DJ, Stelljes M, Martinelli G, Liedtke M, Stock W, et al. Inotuzumab Ozogamicin versus Standard Therapy for Acute Lymphoblastic Leukemia. N Engl J Med. 2016 Aug;375(8):740-53.

24 Lussana F, Intermesoli T, Gianni F, Boschini C, Masciulli A, Spinelli O, et al. Achieving Molecular Remission before Allogeneic Stem Cell Transplantation in Adult Patients with Philadelphia Chromosome-Positive Acute Lymphoblastic Leukemia: Impact on Relapse and Long-Term Outcome. Biol Blood Marrow Transplant. 2016 Nov;22(11):1983-7.

25 Traer E, Javidi-Sharifi N, Agarwal A, Dunlap J, English I, Martinez J, et al. Ponatinib overcomes FGF2-mediated resistance in CML patients without kinase domain mutations. Blood. 2014 Mar;123(10):1516-24.

26 Ueda M, de Lima M, Caimi P, Tomlinson B, Little J, Creger R, et al. Concurrent blinatumomab and donor lymphocyte infusions for treatment of relapsed pre-B-cell ALL after allogeneic hematopoietic cell transplant. Bone Marrow Transplant. 2016 Sep;51(9):1253-5.

27 Lutz C, Massenkeil G, Nagy M, Neuburger S, Tamm I, Rosen O, et al. A pilot study of prophylactic donor lymphocyte infusions to prevent relapse in adult acute lymphoblastic leukemias after allogeneic hematopoietic stem cell transplantation. Bone Marrow Transplant. 2008 May;41(9):805-12.

28 Eefting M, Halkes CJ, de Wreede LC, van Pelt CM, Kersting S, Marijt EW, et al. Myeloablative $\mathrm{T}$ cell-depleted alloSCT with early sequential prophylactic donor lymphocyte infusion is an efficient and safe post-remission treatment for adult ALL. Bone Marrow Transplant. 2014 Feb;49(2):287-91. 